\title{
A Pictorial Description of Steady-States in Rapid Magnetic Resonance Imaging
}

\author{
KLAUS SCHEFFLER \\ MR-Center and Biocenter, University of Basel, Switzerland; E-mail: ksch@ukl.uni-freiburg.de
}

\begin{abstract}
Magnetic resonance imaging in biochemical and clinical research requires rapid imaging sequences. Time-resolved imaging of heart movement and the acquisition of a three-dimensional image block within the circulation time of a contrast agent bolus are two typical examples. Rapid imaging sequences are characterized by a very fast train of radiofrequency (rf) and gradient pulses. Between these rf pulses, the excited magnetization is unable to return to its thermal equilibrium. As a consequence, further rf pulses will influence both the remaining transversal and the remaining equilibrium state. The steady-state magnetization of a multi-rf pulse and gradient pulse experiment is thus a mixture or superposition of different transversal and longitudinal states and the acquired image amplitude becomes a complex function of the investigated tissue's relaxation properties. Based on the works of Woessner, Kaiser, and Hennig, this article intends to give a pictorial description of rapid multipulse imaging experiments. It also provides an extension of this theory applied to modern imaging sequences such as TRUE FISP and rf-spoiled techniques. (c) 1999 John Wiley \& Sons, Inc. Concepts Magn Reson 11: 291-304, 1999.
\end{abstract}

KEY WORDS: rapid imaging; refocusing; steady-state; phase-graph description; rf spoiling

\section{INTRODUCTION}

Most clinically relevant magnetic resonance imaging (MRI) techniques are multipulse techniques which acquire the inverse image space or $\mathbf{k}$-space in a row-by-row sampling pattern. The amplitude of the measured signal defines the image contrast. First, it depends on the intrinsic tissue relaxation times $T_{1}$ and $T_{2}$, and on diffusion effects. Second,

Received 4 January 1999; revised 3 March 1999; accepted 16 March 1999.

Concepts in Magnetic Resonance, Vol. 11(5) 291-304 (1999)

(c) 1999 John Wiley \& Sons, Inc.

CCC 1043-7347/99/050291-14 it is a function of the parameters of the imaging sequence such as the repetition time $T_{R}$, the echo time $T_{E}$, the flip angle of the radiofrequency (rf) excitation pulse $\alpha$, and the gradient waveform design.

It is commonly accepted that a short repetition time of consecutive rf pulses will increase the saturation of longitudinal magnetization, and thus will decrease the amplitude of the free-induction decay (FID) for long $T_{1}$ species. This principle is best described by the well-known Ernst formula (1)

$$
M_{T}=M_{0} \frac{1-E_{1}}{1-E_{1} \cos \alpha} \sin \alpha
$$


which provides an analytical relation between the signal intensity of the FID $\left(M_{T}\right)$ as a function of the relaxation parameter $E_{1}=e^{-T_{R} / T_{1}}$, and the flip angle $\alpha$ by which the initial magnetization $M_{0}$ is moved away from its equilibrium position along the $z$ axis. For a conventional pulse-and-acquired experiment Eq. [1] demonstrates that a rapid excitation of the spin system by intense rf pulses will easily lead to saturation and will drastically reduce the amplitude of the FID.

However, a completely different behavior is observed in almost all types of rapid imaging sequences. This is based on the fact that besides excitation of longitudinal magnetization each $\mathrm{rf}$ pulse will refocus transversal magnetization (like a $\pi$ pulse), which then significantly contributes to the measured signal amplitude.

A typical example is shown in Fig. 1. The stomach of a normal volunteer was filled with $500 \mathrm{~mL}$ water, which has $T_{1}$ and $T_{2}$ values of about $1 \mathrm{~s}$ at $1.5 \mathrm{~T}$. Images were acquired with a gradient echo sequence using a $T_{R}$ of $6 \mathrm{~ms}$ and a flip angle of $70^{\circ}$.

According to the Ernst formula [1] water should be almost completely saturated with these imaging parameters. However, Fig. 1 demonstrates a very intense water signal. This surprising result can be understood by the fact that Eq. [1] is valid only in the case of purely longitudinal interference (1). Tissues with long spin-spin relaxation times $T_{2}$ (compared to $T_{R}$ ) additionally generate a steady state transverse interference: Residual transverse magnetization at the end of the $T_{R}$ interval will be refocused by the next rf pulse and may appear as a spin echo or higher-order echo in the next acquisition window. This influences the signal intensity by constructive or destructive

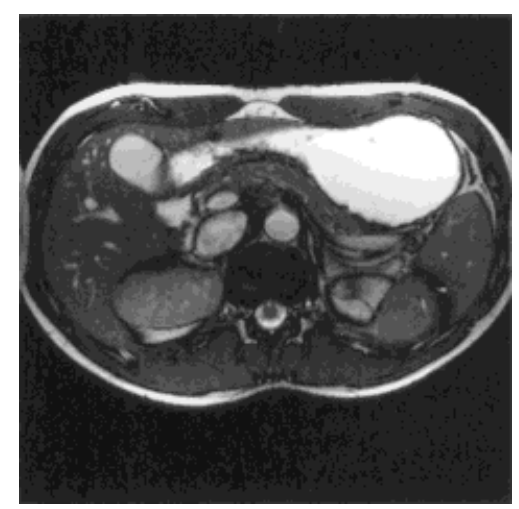

Figure 1 Transaxial slice showing an intense signal of a water-filled human stomach. The image was acquired with a gradient echo sequence (TRUE FISP (14)) using a repetition time $T_{R}$ of $6 \mathrm{~ms}$ and a flip angle of $70^{\circ}$. interferences. Such effects become more significant if $T_{R}$ is about $T_{2}$ of the investigated tissue or even less. Modern state-of-the-art gradient coil and gradient amplifier systems are able to switch all relevant gradients needed to acquire a single row in k-space within 3-5 ms. The boundary condition to investigate the influence of rapid pulse trains on a spin system is therefore given by

$$
T_{R} \ll T_{2}<T_{1}
$$

The analysis of the flow of magnetization from one excitation pulse to the next by means of the Bloch equations is not very helpful for a pictorial understanding. There is no doubt that the Bloch equations can describe the trajectory of each magnetization vector within the imaging plane for an arbitrarily sophisticated imaging experiment. However, this approach provides little insight into the effects of rf pulses and gradient pulses on the spin ensemble, and is not helpful for understanding imaging artifacts or the design of new imaging sequences.

The following article introduces the basics of the phase-graph formalism. This is a very elegant tool with which to understand and calculate the flow of magnetization between consecutive $\mathrm{rf}$ pulses. Based on this formalism, the principles of echo generation and the calculation of echo amplitudes are greatly simplified compared to the direct approach of the application of the Bloch equation separately to each spin.

\section{EXCITATION PULSES AND GRADIENT PULSES}

\section{One pulse}

The basic experiment in Fourier MR is the excitation of the sample (for example, water) by a short $\alpha$ pulse. Relaxation during rf excitation will be neglected throughout this article and the behavior of the magnetization will be discussed exclusively in the rotating $x^{\prime} y^{\prime}$-frame (i.e., the Larmor frequency is equal to the frequency of the receiver synthesizer). The effect of the $\alpha$ pulse is to tip the equilibrium longitudinal magnetization $M_{0}$ toward the transverse $x^{\prime} y^{\prime}$ plane. The resultant magnetization just after an $\alpha$ pulse applied along the $x^{\prime}$ axis is composed of a transversal part $M_{y}=-M_{0} \sin \alpha$ and a remaining longitudinal part $M_{z}=M_{0} \cos \alpha$.

Next, the transversal magnetization of the water sample will dephase, owing to field inhomogeneities, susceptibility effects, or, more importantly, switched imaging gradients. Such a gra- 


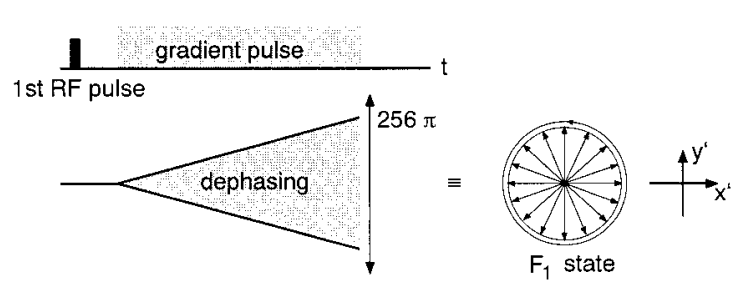

Figure 2 The pure transversal magnetization after the rf excitation pulse (black bar) dephases by the applied gradient pulse (gray bar). For 256 sampling points, the dephasing of the outermost voxel will be $256 \pi$. After the gradient pulse, the magnetization is evenly distributed on a disk in the $x^{\prime} y^{\prime}$ plane, called $F_{1}$ configuration.

dient, for example in the $x$ direction, as shown in Fig. 2, enables the calculation of the projection of the sample in $x$ direction by applying a Fourier transformation to the FID.

In a typical imaging experiment which acquires a $256 \times 256$ matrix, each projection step samples 256 complex data points under the read gradient. According to the sampling theorem, a dephasing by at least 128 full turns will occur to the outermost voxels of the sample. To a very good approximation, this final dephased state can therefore be described by transversal magnetization vectors which are evenly distributed over the transversal plane. The detectable net magnetization at the end of the gradient pulse is zero. This is indicated by the triangle in Fig. 2, depicting the infinite extend of isochromats dephasing at different positions along the $x$ axis.

To analyze multipulse experiments, it is essential to understand in more detail the final state of transversal magnetization. This state is symbolically described by a disk of vectors surrounded by the circular arrow in Fig. 2. The measurable signal is zero because the vector sum of all isochromats is zero. However, the amplitude of each isochromat is still $M_{0} \sin \alpha$, decreased by the factor $e^{-T_{R} / T_{2}}$ due to spin-spin relaxation, which is small for the previously defined condition $T_{R} \ll T_{2}$. For further discussion, it is sufficient to characterize this dephased state by $F_{1}$, where the index 1 stands for one dephasing period induced by the applied imaging gradient.

Several possibilities exist for converting such a dephased transversal state into a measurable net magnetization (or into an echo). A common procedure is to apply a reversed gradient pulse resulting in a complete refocusing of all isochromats to the $y^{\prime}$ axis (gradient echo). Another mechanism to refocus dephased magnetization is the application of a second rf pulse just after the first gradient pulse. A $180^{\circ}$ pulse is known to invert the phase history of all isochromats leading to the spin echo. However, as will be discussed in more detail below, each $\mathrm{rf}$ pulse with an arbitrary flip angle $\alpha$ is able to refocus dephased spins. This was proven already by E. L. Hahn's spin-echo experiment in 1950 , in which the echo was created by two $90^{\circ}$ pulses rather than by a $90^{\circ}-180^{\circ}$ combination (2).

\section{Two pulses}

The pulse timing and the resulting dephased states are shown in Fig. 3. The second $\alpha$ pulse will rotate the transversal configuration $F_{1}$ around the $x^{\prime}$ axis. The result is a disk of magnetization vectors which is tilted by the angle $\alpha$ out of the $x^{\prime} y^{\prime}$ plane. This state, just after the second rf pulse, can be split into a transversal and a longitudinal component. The transversal part, which is a projection of the tilted disk onto the $x^{\prime} y^{\prime}$ plane, has the shape of an ellipse. The longitudinal component consists of different vectors along the $z$ direction and the amplitude of these vectors are given by a sine function. To simplify further discussion, the new transversal component will be divided into more practical configurations.

The elliptic distribution of transversal magnetization can be written as the sum of two dephased states: namely, an $F_{1}^{+}$state and an $F_{-1}^{+}$state. The plus sign indicates the states just after the rf pulses, and the negative index -1 denotes the complex conjugate state of $F_{1}$, i.e., an inversion of the $y^{\prime}$ components of all transversal magnetization vectors. Both states are fully dephased

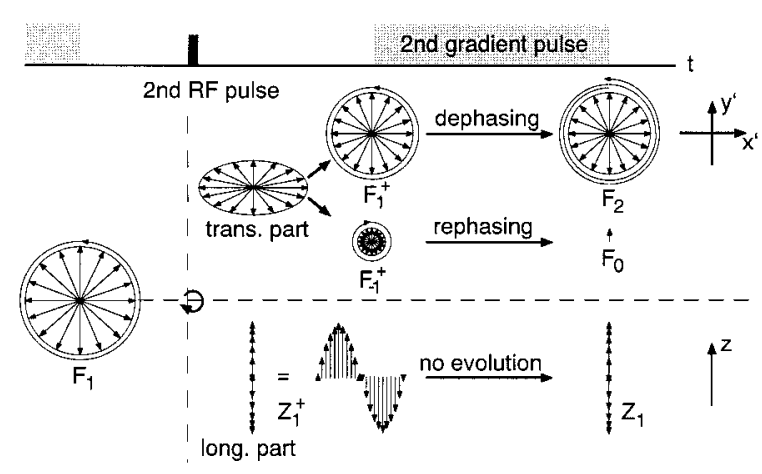

Figure 3 The second rf pulse rotates the transversal $F_{1}$ configuration into a new elliptical transversal state and into a longitudinal state. The new transversal part is a sum of two circularly distributed, transversal states with different amplitudes, $F_{1}^{+}$and $F_{-1}^{+}$. The phase history of these states is reversed to each other and $F_{-1}^{+}$ can thus be rephased by a second gradient pulse into a spin echo $\left(F_{0}\right)$. 
transversal states $\left(F_{-1}^{+}\right.$has a reversed phase history) and the vector sum of the disks will exactly give the ellipse if the amplitudes (or populations) of the states $F_{1}^{+}$and $F_{-1}^{+}$are correctly adjusted. At the moment, the distribution of longitudinal magnetization $Z_{1}^{+}$is not important since it does not evolve in time (it only relaxes by $T_{1}$ effects).

As known from Hahn's experiment, one transverse configuration must exist which can be refocused to form the spin echo. Since the phase history of the new $F_{-1}^{+}$configuration is reversed compared to that of $F_{1}^{+}, F_{-1}^{+}$will refocus exactly at the end of the second gradient pulse (if the first and second gradient pulse have the same areas). This is the spin echo produced by two arbitrary $\alpha$ pulses. The amplitude of this echo is given by the population of the $F_{-1}^{+}$state. It can be seen immediately that a $180^{\circ}$ pulse will transfer all magnetization from the $F_{1}$ state into the $F_{-1}^{+}$state and the population of $F_{1}^{+}$will be zero.

Besides the manipulation of the $F_{1}$ state, the second $\alpha$ pulse will also act on the pure longitudinal magnetization $M_{z}$ (which is decreased to $M_{z}=M_{0} \cos \alpha$ from the first pulse). $M_{z}$ will be flipped into the transversal plane leading to an ordinary FID just after the pulse. This pure transversal magnetization will then be dephased into an $F_{1}$ state at the end of the second gradient pulse. Accordingly, the newly created states $F_{1}^{+}$and $F_{-1}^{+}$will evolve into an $F_{2}$ and an $F_{0}$ (the spin-echo) state, respectively.

\section{Three Pulses}

For the two-pulse experiment, the induced elliptical transversal magnetization was expressed as a sum of two fully dephased, counterrotating $F_{1}^{+}$ and $F_{-1}^{+}$states. The double-dephased $F_{2}$ state at the end of the second gradient pulse in Fig. 3 will be converted again into an elliptical configuration by the third pulse, which then can be separated again into an $F_{2}^{+}$and $F_{-2}^{+}$state.

The sine-shaped distribution of longitudinal magnetization $Z_{1}$ will be tipped into the transversal plane by the third pulse as shown in Fig. 4 . The third $\alpha$ pulse will create a transversal configuration where all tips of the magnetization vectors are aligned in $\pm y^{\prime}$ direction and a remaining, reduced longitudinal configuration $Z_{1}^{+}$.

The net magnetization of the new transversal configuration is zero, but a certain fraction can be refocused by the gradient pulse into a stimulated echo. Once more, the trick is to decompose the aligned vectors into a pair of dephased states, one

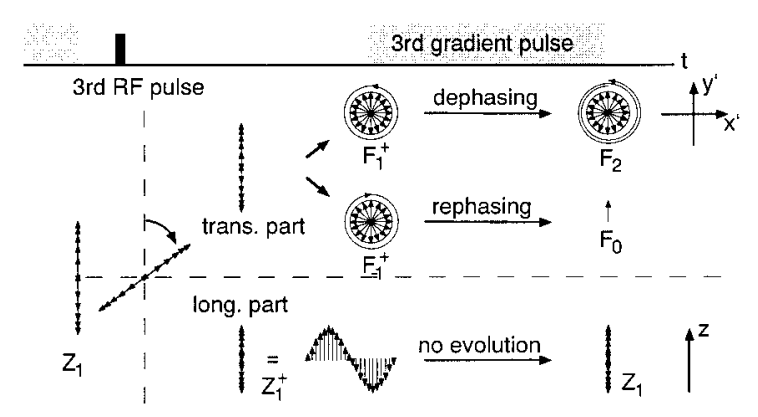

Figure 4 The third rf pulse transfers the longitudinal $Z_{1}$ configuration into a transversal part and a remaining longitudinal part. Both configurations consist of sinusodial alignments of magnetization vectors along $y^{\prime}$ and $z$ direction. The transversal part can be split into two circular configurations, $F_{1}^{+}$and $F_{-1}^{+}$, one being the complex conjugate of the other. $F_{-1}^{+}$is refocused to the stimulated echo $\left(F_{0}\right)$ after the third gradient pulse.

being the complex conjugate of the other. These states have the same amplitudes (or same diameters of the circle); otherwise their vector sum would create an ellipse as demonstrated in Fig. 3, and not, a sum of vectors aligned in the $\pm y^{\prime}$ direction. This decomposition is equivalent to the description of a linearly polarized wave as the sum of two counterrotating, circularly polarized waves. The phase history of these states was created by the first gradient pulse, and we thus can call them $F_{1}^{+}$and $F_{-1}^{+}$. The $F_{-1}^{+}$state will be refocused by the third gradient pulse to form the stimulated echo, described as $F_{0}$ in Fig. 4. A negative, third gradient pulse would force $F_{1}^{+}$to refocus as a stimulated echo.

All relevant mechanisms have now been described to understand multiple-pulse experiments. All possible distributions of transversal magnetization vectors can be decomposed into $F_{n}$ and $F_{-n}$ states, where $n$ indicates the phase history of the configuration (the number of gradient pulses exposed to the configurations). Possible distributions of longitudinal magnetization are characterized by sine-shaped alignment of vectors in the $z$ direction (the $z$ components of the tilted disk in Fig. 3). They are called $Z_{n}$ states, depending on the previous $F_{n}$ state.

However, the representation of all possible $Z_{n}$ and $F_{n}$ states by magnetization vectors distributed on disks or aligned along $z$ direction becomes very complex for an increasing number of rf pulses. A more practical and pictorial description of the creation and flow of states from one excitation pulse to the next offers the extended phase-graph technique based on the works of Kaiser et al. (4) and Hennig (5). 


\section{THE PHASE-GRAPH DESCRIPTION}

\section{Transfer and Evolution of States}

An easy and illustrative way to display the creation and evolution of the $F_{n}, F_{n}^{*}$, and $Z_{n}$ states is the two-dimensional phase-graph technique (5). Figure 5 describes the stimulated echo experiment $(2,6)$ and gives a pictorial description of the origin of the different echoes beside the stimulated echo. The creation of new states is indicated by small arrows which connect corresponding pre- and postpulse states-for example, $F_{1}$ with $F_{1}^{+}, F_{-1}^{+}$, and $Z_{1}^{+}\left(Z_{n}^{+}\right.$states appear as broken lines) for the second rf pulse. In this example, the area of the gradient pulses and the rf pulse spacing is not constant. This allows the observation of all echo paths. The dephasing or the gradient pulse area between the first and second rf pulse is 1 , and is 4 between the second and third rf pulse. A primary spin echo is formed between the first and second rf pulse by a refocused $F_{-1}^{+}$state, which is indicated by a small circle (it goes without saying that we do not need a $180^{\circ}$ pulse for refocusing). The position of the echo coincides with the zero-crossing of the $F_{-1}^{+}$ line, which is then an $F_{0}$ state showing measurable, pure magnetization in the $y^{\prime}$ direction.

If the second gradient pulse had a negative polarity, an echo would be created by the refocusing of the $F_{1}^{+}$state and $F_{-1}^{+}$would further dephase into $F_{-5}$. However, the $F_{1}^{+}$state was not created by the refocusing mechanism of the second rf pulse. Thus, in the case of a negative second gradient pulse, the echo should be actually called a gradient echo, however, with reduced intensity compared to a conventional gradient echo. This is because parts of the $F_{1}$ state are transferred into $F_{-1}^{+}$and $Z_{1}^{+}$states.

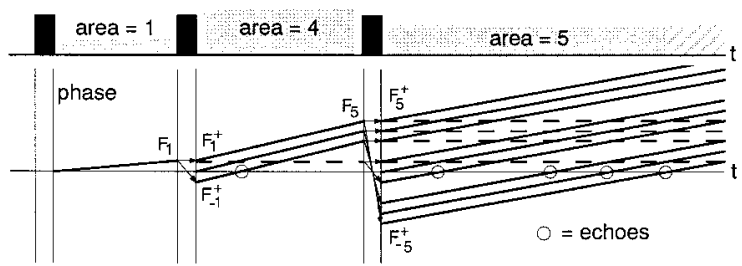

Figure 5 Five echoes can be generated with a three pulse experiment. A spin echo appears after the second rf pulse owing to the refocused $F_{-1}^{+}$component. The $F_{1}^{+}, F_{0}^{+}$, and $F_{-1}^{+}$configurations after the second rf pulse will then dephase into $F_{1+4}, F_{0+4}$, and $F_{-1+4}$ configurations. The third rf pulse converts these configurations into nine new $F_{n}$ configurations, but only $F_{-5}^{+}, F_{-4}^{+}, F_{-3}^{+}$, and $F_{-1}^{+}$will refocus into a stimulated echo and higherorder echoes. Note the varying slope of the phase graph according to the different gradient amplitudes.
The second gradient pulse will dephase $F_{1}^{+}$into $F_{5}, F_{0}^{+}$into $F_{4}, F_{-1}^{+}$into $F_{3}$, and $Z_{1}^{+}$remains constant and is called $Z_{1}$ before the third rf pulse. The third rf pulse partially converts the $F_{n}$ states into $F_{-5}^{+}, F_{-4}^{+}, F_{-3}^{+}$, and $Z_{1}$ into $F_{1}^{+}$and $F_{-1}^{+}$. The last gradient pulse rephases these configurations into a stimulated echo for $F_{-1}^{+}$, and spin echoes for $F_{-3}^{+}, F_{-4}^{+}$, and $F_{-5}^{+}$. In the case of equidistant rf pulses and identical gradient areas the stimulated echo and the third spin echo will collapse into one echo. Depending on the phases of the $\mathrm{rf}$ pulses (which will be investigated in detail later), these echoes can add up either constructively or destructively.

It is important to note that the zero-crossing of the phase graph coincides with the position of the center of the corresponding echo. Depending on the strength of the readout gradient, the detected echo signal extends to the left and right of the zero-crossing. This can lead to interference between neighboring echoes; a practical application of this phenomenon is MR interferography to detect flow or motion (7).

The phase graph of the stimulated echo experiment is still valid in the presence of $B_{0}$ inhomogeneities. A $B_{0}$ offset during the three pulse experiment just shifts the base frequency of each isochromat; the echo positions will be unaffected. The last missing tool for a full description of multipulse experiments is the calculation of the amplitudes of the echoes, or, equivalently, the population of all possible $F_{n}$ and $Z_{n}$ states.

\section{Population of the States}

The calculation of echo amplitudes closely follows the formalism given by Jaynes and Woessner $(8,3)$. Introducing the complex magnetization $F=M_{x}+i M_{y}$, the effect of an rf pulse with flip angle $\alpha$ and phase $\phi$ is equivalent to a vector rotation. It can be expressed by the complex Bloch equation

$$
\begin{aligned}
F^{+}= & \cos ^{2}\left(\frac{\alpha}{2}\right) F+e^{2 i \phi} \sin ^{2}\left(\frac{\alpha}{2}\right) F^{*} \\
& -i e^{i \phi} \sin (\alpha) M_{z} \\
M_{z}^{+}= & -\frac{i}{2} e^{-i \phi} \sin (\alpha) F+\frac{i}{2} e^{i \phi} \sin (\alpha) F^{*} \\
& +\cos (\alpha) M_{z}
\end{aligned}
$$

where $F^{*}$ is the complex conjugate of $F$. These equations describe how rf pulses act on pure magnetizations. 
However, we are interested in the effect of $\mathrm{rf}$ pulses on fully dephased $F_{n}$ states and longitudinal $Z_{n}$ states. An $F_{n}$ state is a sum over dephased spins located at different spatial positions. The phase evolution of spins at position $\mathbf{r}$ induced by $n$ identical gradient lobes is $e^{i n \boldsymbol{\phi}_{\mathbf{G}} \mathbf{r}}$. $\boldsymbol{\phi}_{\mathbf{G}}$ is the area of the gradient pulses switched between the rf pulses and it is given by $\gamma \mathbf{G T}$, where $\mathbf{G}$ is the gradient amplitude in $\mathrm{T} / \mathrm{m}$ and $T$ the duration of the gradient pulse ${ }^{1}$. An $n$-fold dephased transversal state can thus be written as a sum or integral over all positions

$$
\text { transversal state }=F_{n} \int_{\mathbf{r}} e^{i n \boldsymbol{\phi}_{\mathrm{G}} \mathbf{r}} d \mathbf{r}
$$

The magnitude of the complex number $F_{n}$ is the amplitude or population of this state and the phase of $F_{n}$ is the angle in the $x^{\prime} y^{\prime}$ plane. Accordingly, the $Z_{n}$ state, which consists of a sinusodial and cosinusodial distribution of magnetization along the $z$ direction, can be expressed as

$$
\text { longitudinal state }=Z_{n} \int_{\mathbf{r}} e^{i n \boldsymbol{\phi}_{\mathrm{G}} \mathbf{r}} d \mathbf{r}
$$

As shown in the previous examples, the magnetization after several rf pulses is a sum of different dephased states $F_{n}$. This can be expressed as

$$
\begin{aligned}
& F=\sum_{n=-\infty}^{\infty} F_{n} \int_{\mathbf{r}} e^{i n \boldsymbol{\phi}_{\mathbf{G}} \mathbf{r}} d \mathbf{r} \\
& Z=\sum_{n=-\infty}^{\infty} Z_{n} \int_{\mathbf{r}} e^{i n \boldsymbol{\phi}_{\mathbf{G}} \mathbf{r}} d \mathbf{r}
\end{aligned}
$$

Since longitudinal magnetization is a real value, it follows that $Z_{n}=Z_{-n}^{*}$. Inserting these equations into the complex Bloch equation [3] will finally give

$$
\begin{aligned}
& \left(\begin{array}{c}
F_{n} \\
F_{-n}^{*} \\
Z_{n}
\end{array}\right)^{+} \\
& =\left(\begin{array}{ccc}
\cos ^{2} \frac{\alpha}{2} & e^{2 i \phi} \sin ^{2} \frac{\alpha}{2} & -i e^{i \phi} \sin \alpha \\
e^{-2 i \phi} \sin ^{2} \frac{\alpha}{2} & \cos ^{2} \frac{\alpha}{2} & i e^{-i \phi} \sin \alpha \\
-\frac{i}{2} e^{-i \phi} \sin \alpha & \frac{i}{2} e^{i \phi} \sin \alpha & \cos \alpha
\end{array}\right) \\
& \\
& \quad\left(\begin{array}{c}
F_{n} \\
F_{-n}^{*} \\
Z_{n}
\end{array}\right)
\end{aligned}
$$

${ }^{1} \mathbf{G}$ is composed of the three Cartesian components $\left(G_{x}, G_{y}, G_{z}\right)$. For time-varying gradient pulses $\mathbf{G}(t)$, the gradient area is given by $\boldsymbol{\phi}_{\mathbf{G}}=\gamma \int_{0}^{T} \mathbf{G}(t) d t$.
This expression describes the transition from prepulse to postpulse states for each $n$. The spatial integrals over $\mathbf{r}$ in Eqs. [4] and [5] are canceled since the transition rule applies for all positions r. Inspection of the matrix equation [7] yields an exact mathematical analogy to the phase-graph representation in Fig. 5: Each rf pulse creates three magnetization components: two transversal states with complementary phase evolution $\left(F_{n}, F_{-n}\right)$ and one longitudinal state $\left(Z_{n}\right)$. The small arrows between pre- and postpulse states shown in Fig. 5 are graphically analogous to the transition matrix [7].

In addition, we need a rule for the evolution of states between $\mathrm{rf}$ and gradient pulses. Beside CPMG-type sequences (18), all rapid imaging sequences are multipulse experiments with constant rf pulse spacing and constant net dephasing between rf pulses. A constant net dephasing requires constant readout and slice selection gradient pulses and a balanced or rewound phase-encoding gradient. The schematic timing diagram, partially adapted from (16), is depicted in Fig. 6. Detailed readout and phase encoding gradient lobes are shown in Figs. 8 and 9. Since the amount of dephasing is restricted to multiples of one readout block, the creation and evolution of different $F_{n}$ and $Z_{n}$ states is strongly restricted.

According to Fig. 6 , the evolution of states between rf pulses is given by:

$$
\left.\begin{array}{ccc}
F_{n}^{+} \longrightarrow & \begin{array}{c}
F_{n+1}=E_{2} F_{n}^{+} \\
\text {dephasing and } T_{2} \text { relaxation }
\end{array} \\
Z_{n}^{+} \longrightarrow & \begin{array}{l}
Z_{n}=E_{1} Z_{n}^{+} \\
\text {for } n \neq 0
\end{array} \\
Z_{0}^{+} \longrightarrow & \begin{array}{l}
Z_{0}=E_{1} Z_{0}^{+} \\
\\
+
\end{array} \\
& M_{0}\left(1-E_{1}\right)
\end{array}\right\} T_{1} \text { relaxation }
$$

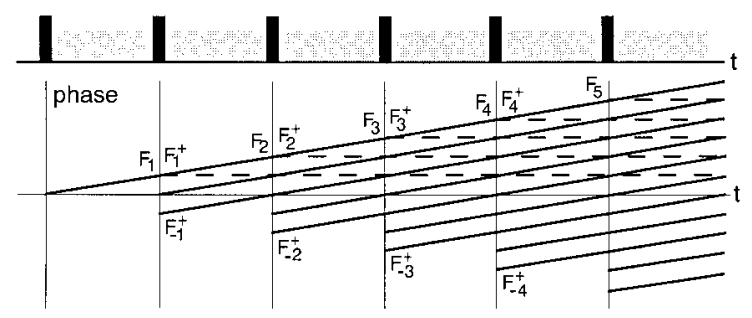

Figure 6 Basic template of a multipulse imaging sequence. Between consecutive rf pulses (black bars), the gradient pulses (gray bars) dephase the transversal $F_{n}^{+}$ states into $F_{n+1}$ states. Since all gradient pulses have the same area $\gamma G T$, the generation of new states increases linearly with increasing rf pulse number. After several rf pulses, the pure transversal magnetization $F_{0}^{+}$becomes a sum of refocused $F_{-n}$ states and the FID. 
The parameters $E_{1}=e^{-T_{R} / T_{1}}$ and $E_{2}=e^{-T_{R} / T_{2}}$ attenuate these configurations owing to $T_{1}$ and $T_{2}$ relaxation effects.

Again, the rules in Eq. [8] cannot be applied to varying gradient pulses and rf pulse spacings, as shown in the stimulated echo example in Fig. 5. For equally spaced pulses (and constant gradient integrals in between), the number of states increases linearly (Fig. 6) but can grow exponentially in the worst case $(15,19)$.

As an exercise, we calculate the amplitude of a spin echo generated by two rf pulses $\alpha_{1}$ and $\alpha_{2}$ around the $x^{\prime}$ axis $(\phi=0)$. Starting with equilibrium magnetization $M_{0}=M_{z}=Z_{0}=1$ the magnetization after the first pulse will be $F_{0}^{+}=$ $-i \sin \alpha_{1}, F_{0}^{+*}=i \sin \alpha_{1}$, and $Z_{0}^{+}=\cos \alpha_{1}$. This corresponds to pure transversal magnetization in $-y^{\prime}$ direction with amplitude $\sin \alpha_{1}$ and a remaining longitudinal magnetization of $\cos \alpha_{1}$. According to the dephasing and relaxation rules given in Eq. [8], $F_{0}^{+}$will evolve into $E_{2} F_{1}=-i E_{2} \sin \alpha_{1}$ and $F_{0}^{+*}$ into $E_{2} F_{1}^{*}=i E_{2} \sin \alpha_{1}$, all other transversal states are zero. Inserting this into the transition matrix [7] for the second rf pulse $(n=1)$ gives

$$
\begin{aligned}
F_{1}^{+} & =i E_{2} \cos ^{2} \frac{\alpha_{2}}{2} \sin \alpha_{1} \text { and } \\
F_{-1}^{+*} & =E_{2} \sin ^{2} \frac{\alpha_{2}}{2} \sin \alpha_{1}
\end{aligned}
$$

i.e., the population of $F_{-1}^{+}$which forms the echo is $E_{2} \sin ^{2} \frac{\alpha_{2}}{2} \sin \alpha_{1}$. For the conventional spin echo experiment with $\alpha_{1}=\pi / 2$ and $\alpha_{2}=\pi$ the result would be $1.0 \cdot E_{2}$. For the Hahn spin echo with $\alpha_{1}=\alpha_{2}=\pi / 2$ the resulting echo amplitude will be $0.5 \cdot E_{2}$.

\section{Toward the Steady State}

In a typical imaging experiment, 200-500 consecutive excitation pulses and readout gradient pulses (several thousand for three-dimensional encoding) are used to acquire the k-space data. After $N$ pulses the state of the spin ensemble can be described by $2 N+1 F_{n}$ and $N+1 Z_{n}$ configurations. The population of these states depends on the flip angle $\alpha$ and phase $\phi$ of the rf pulses and on the relaxation times $T_{1}$ and $T_{2}$. Under certain conditions that are listed below, a steady state of all configurations can be reached. Constant echo amplitudes during the imaging experiment are essential to obtain artifact-free images, since any modulations during $\mathbf{k}$-space encoding can produce ghosting or interference effects.

The formulas given in Eqs. [7] and [8] can be used to calculate or to simulate the evolution of states for a multipulse experiment. The corresponding $\mathrm{C}$ program is listed in the Appendix. An example for the evolution of $F_{n}$ states is shown in Fig. 7 for the parameters $T_{R}=0.025 \cdot T_{1}=$ $0.05 \cdot T_{2}$, and flip angle $\alpha=70^{\circ}$. The gray values on the left panel are proportional to the populations or amplitudes of the $F_{n}$ configurations. The
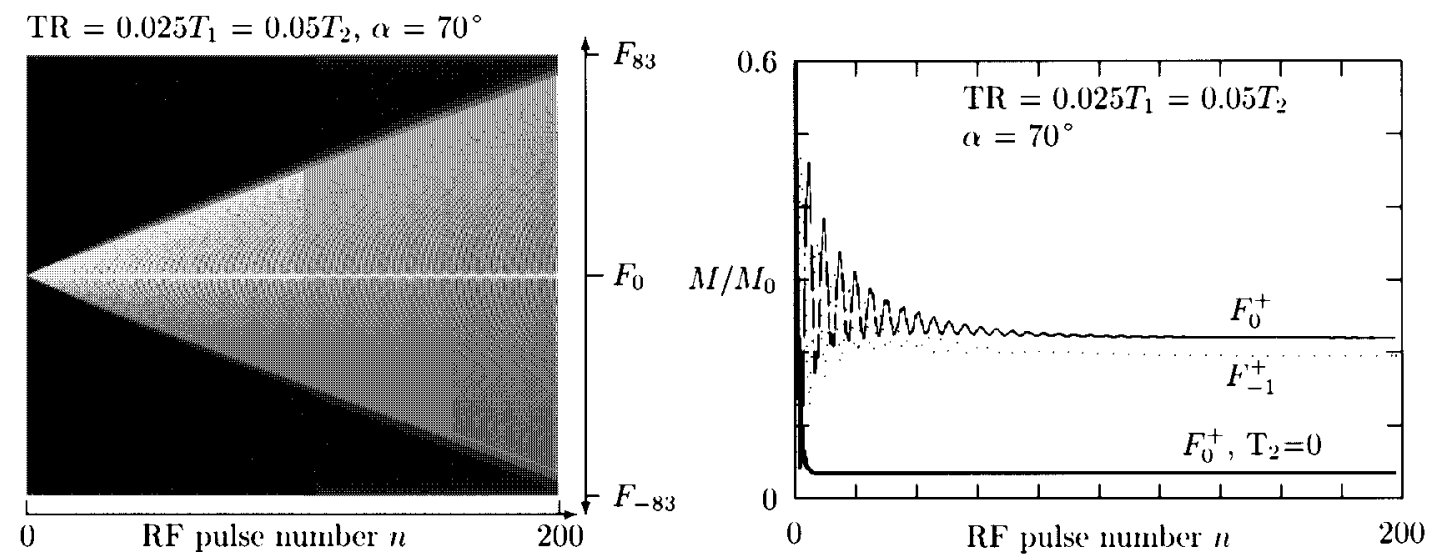

Figure 7 (Left) Generation and flow of transversal $F_{n}$ states during a multipulse experiment. The gray values are proportional to the magnitude of $F_{n}$. The spreading velocity of the peripheral wave depends on the flip angle $\alpha$ of the rf pulse. The refocusing mechanism is dominant for high flip angles and thus results in a small spreading velocity. For low flip angles, most of the magnetization flows to the higher-order $F_{n}$ states. (Right) Time course of the $F_{0}^{+}$and $F_{-1}^{+}$state as a function of the rf pulse number. The strong amplitude oscillation at the beginning of the rf pulse train converges to a steady state after about $100 \mathrm{rf}$ pulses. The final steady-state echo amplitudes are significantly stronger compared to the Ernst amplitude $\left(F_{0}^{+}\right.$for $T_{2}=0$ ). 
right plot in Fig. 7 depicts the amplitude course of the $F_{0}^{+}$state (pure transversal magnetization after the rf pulse) as a function of the rf pulse number $n$. The curve at the bottom also shows the evolution of the $F_{0}^{+}$state for $T_{2}$ being equal to zero. For this case, no transversal magnetization can be refocused and thus does not contribute to subsequent $F_{n}$ states. Therefore, the steady-state amplitude coincides with the Ernst formula [1] for purely longitudinal interference $F_{0}\left(T_{2}=0\right)=$ 0.0348 . The dotted line shows the population of the $F_{-1}^{+}$state which can be converted into a measurable gradient echo by a reversed readout gradient lobe, as described in the next section.

A reduction of the $\mathrm{rf}$ flip angle $\alpha$ will attenuate the refocusing mechanism of the rf pulse. According to Eq. [7], a flip angle of $\alpha=20^{\circ}$, for example, will excite $\sin \left(20^{\circ}\right)=34 \%$ of the longitudinal magnetization, but only $\sin ^{2}\left(20^{\circ} / 2\right)=3 \%$ of the transversal magnetiztion will be refocused. Therefore, the steady-state amplitude is very close to the Ernst amplitude for low flip angles, but differs significantly for high flip angles and long spin-spin relaxation times $T_{2}$.

The oscillating signal amplitudes at the beginning of the rf pulse train relax into the steady state after roughly $5 \cdot T_{2} / T_{R}$ excitation pulses. In most clinical and biological applications, the steady state will be reached after about 50-100 rf pulses $\left(T_{2} \sim 100-200 \mathrm{~ms}\right.$ at $\left.1.5 \mathrm{~T}, T_{r} \sim 10 \mathrm{~ms}\right)$ or even less for small flip angles $\left(\alpha \sim 10^{\circ}-20^{\circ}\right)$. Since the first acquisitions usually correspond to the outermost part of k-space, imaging artifacts are strongly suppressed. High flip angles prolong the approach to constant amplitudes and can produce ringing or ghosting artifacts in the image. One common approach to suppress these effects is a set of prescans to reach equilibrum before data acquisition, or using variable flip angles during the start of acquisition (20). In the next section, an analysis of the population of the configurations after having reached the steady state is made and an analytical expression of their amplitude as a function of $T_{1}, T_{2}$, and flip angle $\alpha$ is given.

\section{THE STEADY STATE}

Until now it has not been discussed whether a steady state of magnetization can be reached. Under certain assumptions, it is easy to obtain some rules that guarantee an approach to equilibrum. However, it is also straightforward to generate some kind of limit cycles or even noise in the magnetization using, for example, stochastic rf phases or flip angles.

\section{Necessary Conditions to Reach the Steady State}

Since most samples are composed of different compartments, the signal amplitudes have to reach the steady state for any given relaxation times $T_{1}$ and $T_{2}$. From this, it follows immediately that the flip angle $\alpha$ of the rf pulses and the rf pulse spacing $T_{R}$ must be constant. In addition, the dephasing of spins during $T_{R}$ must be constant (or zero) to ensure proper refocusing and establish a stationary transversal magnetization. The remaining free parameter is the phase of the rf pulse, meaning the angle of the rotation axis in the $x^{\prime} y^{\prime}$ plane. Equation [7] shows that a phase offset $\phi$ of the rf pulse will rotate all transversal states by $2 \phi$ around the $z$ axis, which then results in a destructive interaction of different states.

It is remarkable and very useful to modify the contrast behavior of the sequence, that the phase of the rf pulse can vary quadratically as a function of the pulse number without disturbing the stationary state. In summary, the following conditions are sufficient and necessary to produce stationary states:

- The dephasing of states between rf pulses must be constant.

- The time $T_{R}$ between rf pulses must be constant.

- The flip angle $\alpha$ of rf pulses must be constant.

- The phases of the rf pulses must satisfy the equation: $\phi_{n}=a+b n+c n^{2}$.

A rigorous proof of these conditions can be found in Sobol et al. (17) and Zur et al. (21).

\section{Steady-State Amplitudes of the Transversal Configurations: Three Approaches}

First Approach. Biological tissues show a large and specific variety in $T_{1}$ and $T_{2}$ relaxation times. In terms of clinical applications and tissue characterization, it is important to know the image contrast as a function of these relaxation parameters. Equation [7] gives the recipe to calculate the amplitudes of all configurations $F_{n}$ and $Z_{n}$ after $n$ excitation pulses. Application of the rf pulse matrix and evolution of states given by Eq. [8] finally 
gives the steady-state amplitudes after sufficient iteration steps; an example is shown in Fig. 7 for the $F_{0}^{+}$and $F_{-1}^{+}$state.

If we are not interested in the evolution of all states starting from the first rf pulse (which, however, gives an excellent idea of the working mechanism of the sequence), two further approaches are derived to calculate the stationary amplitudes.

Second Approach. Under the assumptions that a steady state has been reached and that all phases of the rf pulses are constant, it can be shown that the complex transversal magnetization $F=M_{x}+$ $i M_{y}$ is given by $(22-24)$

$$
\begin{aligned}
F\left(t, T_{1}, T_{2}, \alpha\right) \\
=M_{0} \frac{\left(1-E_{1}\right) \sin \alpha e^{-t / T_{2}}}{p-q \cos \phi_{G}(t)} \\
\cdot\left(1-E_{2} e^{-i \phi_{G}(t)}\right)
\end{aligned}
$$

where

$$
\begin{aligned}
& p=1-E_{1} \cos \alpha-E_{2}^{2}\left(E_{1}-\cos \alpha\right) \\
& q=E_{2}\left(1-E_{1}\right)(1+\cos \alpha)
\end{aligned}
$$

$\phi_{G}(t)$ is the accumulated precession angle or the dephasing over the gradient pulse period $T_{R}$, and $t$ denotes the time evolution of $F$ between two rf pulses $\left(t=0 \cdots T_{R}\right)$. The denominator $1 /(p-$ $\left.q \cos \phi_{G}\right)$ can be expanded into a geometrical series by grouping of echoes with equal phase evolution $\phi_{G}(t)$ and Eq. [10] can be written as

$$
\begin{aligned}
F\left(t, T_{1}, T_{2}, \alpha\right)= & M_{0} \frac{\left(1-E_{1}\right) \sin \alpha e^{-t / T_{2}}}{p} \\
& \cdot \sum_{n=-\infty}^{\infty}\left(u_{n}-E_{2} v_{n}\right) F_{n}
\end{aligned}
$$

with

$$
\begin{aligned}
& u_{n}=\sum_{m=0}^{\infty}\left(\begin{array}{c}
2 m+n \\
m
\end{array}\right)\left(\frac{q}{2 p}\right)^{2 m+n} \\
& v_{n}=\sum_{m=0}^{\infty}\left(\begin{array}{c}
2 m+n+1 \\
m
\end{array}\right)\left(\frac{q}{2 p}\right)^{2 m+n+1}
\end{aligned}
$$

Equations [12] and [13] are a more compact version of the original expression of Gyngell and Mizumoto et al. $(11,12)$ using the convention that $\left(\begin{array}{l}n \\ k\end{array}\right)=0$ for $0 \leq n<k$, and by identifying $e^{i n \phi_{G}(t)}$ with $F_{n}$. The amplitudes just after the $\mathrm{rf}$ pulse $\left(F_{n}^{+}\right.$states) are given for $t=0$ in Eq. [12] and amplitudes just before rf excitation by setting $t=T_{R}\left(F_{n}\right.$ states $)$. Since we are in the steady state we have an infinite amount of $F_{n}$ states ranging from $n=-\infty$ to $\infty$. The amplitudes are given by $u_{n}-E_{2} v_{n}$, and we thus have an analytical relation between $F_{n}$ amplitudes and $T_{1}, T_{2}$, and flip angle $\alpha$. Numerical evaluation of the sums in Eq. [13] exactly gives identical $F_{n}$ amplitudes as for the iterative result using the first approach based on the transition matrix.

Third Approach. A third and equivalent result using a slightly different technique was found by Zur et al. for the stationary amplitudes of the $F_{0}$ and $F_{-1}$ state (13). By integrating $\phi_{G}(t)$ in Eq. [10] over a full $2 \pi$ period, they proved that the amplitude can be expressed as

$$
\begin{aligned}
F_{0}= & \frac{\sin \alpha}{1+\cos \alpha}\left(1-E\left(E_{1}-\cos \alpha\right)\right) \\
F_{-1}= & -\frac{\sin \alpha}{E_{2}(1+\cos \alpha)} \\
& \cdot\left(1-E\left(1-E_{1} \cos \alpha\right)\right)
\end{aligned}
$$

where $E$ is given by

$$
\begin{aligned}
\left(1-E_{2}^{2}\right)^{\frac{1}{2}}\left[1-E_{1}^{2} E_{2}^{2}\right. & -2 E_{1}\left(1-E_{2}^{2}\right) \cos \alpha \\
+ & \left.\left(E_{1}^{2}-E_{2}^{2}\right) \cos ^{2} \alpha\right]^{-\frac{1}{2}}
\end{aligned}
$$

Again, the result coincides with the transfer matrix technique and the approach of Gyngell and Mizumoto et al. Apparently, Mizumoto et al. derived a closed expression for the population of all $F_{n}$ configurations in the steady state. However, the Mizumoto et al. and the Zur et al. approach do not handle the case of quadratically increasing phases of the rf pulses. It is still best calculated by the iterative phase-graph description.

\section{RAPID STEADY-STATE IMAGING SEQUENCES}

Rapid imaging sequences or steady-state free precession (SSFP) sequences acquire k-space data in a steady state of all transversal and longitudinal components. The conditions for reaching the stationary state are known, as well as the relationship between the amplitudes of transversal coherences and the tissue parameters $T_{1}$ and $T_{2}$, and the flip angle $\alpha$. Keeping these rules in mind, a gradient 
switching pattern can be designed to select one of the $F_{n}$ states for data acquisition. The resulting image contrast can be calculated by one of the three techniques described above.

\section{$F_{0}$ and $F_{-1}$ Sequences}

Most rapid steady-state sequences use the $F_{0}$ or $F_{-1}$ state for k-space acquisition. The corresponding timing diagrams are shown in Fig. 8. The acronyms for these sequences are FAST, GRASS, or FISP (9) (upper panel), and CE-FAST or PSIF (10) (lower panel). It is obvious that the phaseencoding gradient (one for two-dimensional and two for three-dimensional imaging) must be rewound prior to the next rf pulse. Otherwise, the total dephasing of spins (or the gradient area) between rf pulses is not constant and thus will disturb the steady state. In both sequence types, the net dephasing between pulses is equal and the distribution of magnetization onto the $F_{n}$ states is equivalent.
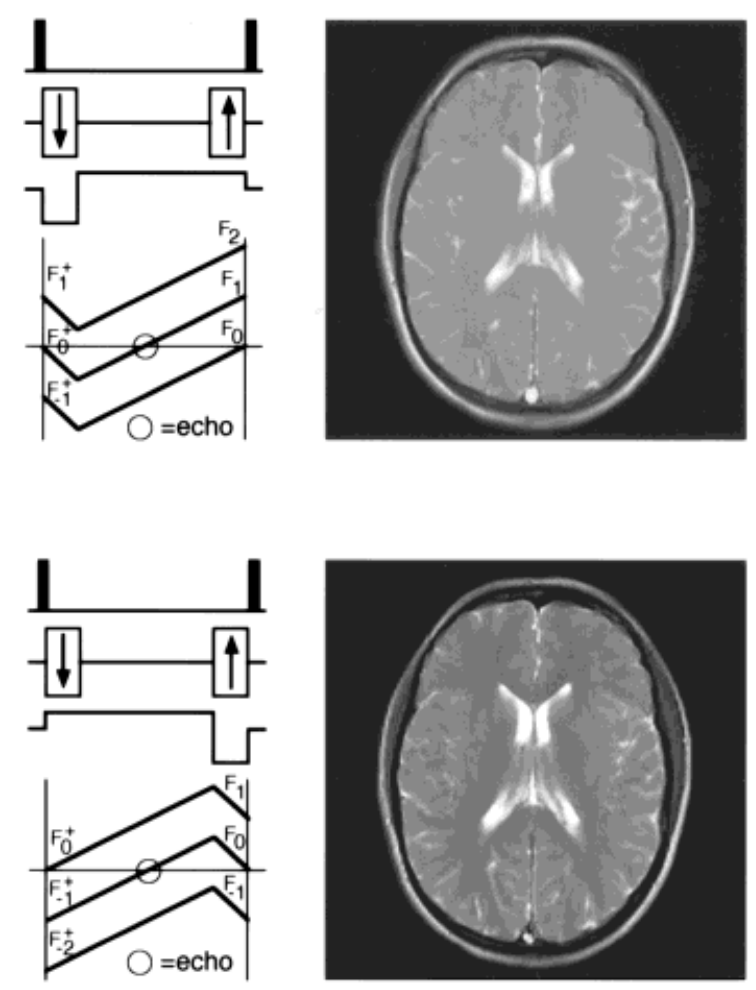

Figure 8 (Top) FAST, GRASS, or FISP sequence. (Bottom) CE-FAST or PSIF sequence. Compared to the FAST sequence, the readout gradient of CE-FAST is time reversed. $F_{0}^{+}$will be refocused to the acquisition echo for the FAST sequence and $F_{-1}^{+}$for the CE-FAST sequence. The CE-FAST image is stronger $T_{2}$-weighted compared to the FAST image, since $F_{-1}^{+}$is mainly composed of spin echo signals.
The particular lobe of the readout gradient determines which $F_{n}$ state is selected for imaging, yielding different image contrasts as shown in the corresponding images in Fig. 8. The cerebrospinal fluid (CSF) has $T_{1}$ and $T_{2}$ values of about 1200/200 ms, and white matter has a $T_{1}$ and $T_{2}$ of about $500 / 70 \mathrm{~ms}$ at $1.5 \mathrm{~T}$. For the imaging parameters of $\alpha=40^{\circ}$ and $T_{R}=11 \mathrm{~ms}$, it follows from Eqs. [14-16] that the CE-FAST sequence shows an increased ratio between CSF and white-matter signal corresponding to a $T_{2}$-like contrast. A smaller flip angle will reduce transversal refocusing and thus will significantly decrease the signal amplitude of the long $T_{2}$ CSF.

\section{$F_{|n|>1}$ Sequences}

Beside the distinct difference in the contrast behavior of higher-order $F_{n}$ sequences $(|n|>1)$, a significant drawback is the strongly reduced intensity of these states compared to the most intense $F_{0}$ state. Numerical evaluation of the power series expansion given in Eq. [13] shows that the amplitudes of $F_{n}$ states decrease exponentially as a function of $n$. Since noise in nuclear magnetic resonance (NMR) experiments does not decrease, the signal-to-noise ratio for high-order $F_{n}$ sequences is drastically reduced. Figure 9 shows a sequence which selects the $F_{-2}$ path for imaging. The parameters used in this experiment were $\alpha=40^{\circ}$ and $T_{R}=15 \mathrm{~ms}$. Figure 9 demonstrates an increased signal ratio between long and short $T_{2}$ tissues compared to the FAST or CE-FAST sequence, since the contribution of high-order echoes to the image signal is emphasized for the $F_{-2}$ path.
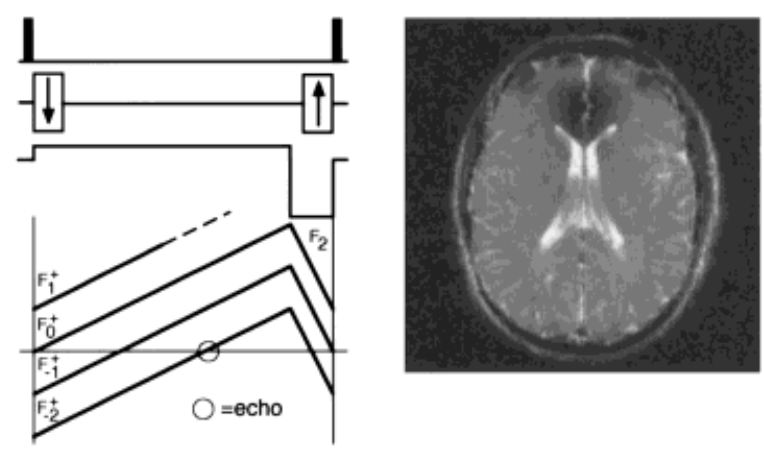

Figure 9 The long readout gradient refocuses both the $F_{-1}^{+}$and $F_{-2}^{+}$state, but only the second echo is acquired for image formation. The resulting image shows a reduced signal-to-noise ratio compared to the previous examples. In addition, through-plane dephasing artifacts are visible at the frontal region of the brain induced by local field inhomogeneities. 


\section{Rf Spoiling: Ernst Angle Revisited}

The previous examples are based on rf pulses with a constant phase angle. This results in a constructive addition of $F_{n}$ states and the amplitude of the stationary $F_{0}$ state is much higher than would be expected from pure longitudinal interferences (Ernst formula [1], $E_{2}=0$, or no refocusing of transversal components). As first described by Zur et al. (21), a quadratic increment of the rf pulse phases can produce destructive interferences between different states, and in the ideal case only the pure FID after the rf pulse contributes to the image signal. The resulting $T_{1}$-weighted imaging sequences are of great importance in clinical practice: for example, in displaying vessels in contrastenhanced angiography techniques (25).

Figure 10 shows the intensity of the $F_{0}^{+}$signal for different quadratic phase increments $c\left(\phi_{n}=\right.$ $\left.\frac{n(n-1)}{2} c\right)$, and for $T_{R}=0.02 \cdot T_{1}=0.02 \cdot T_{2}$ and $\alpha=60^{\circ}$. The dotted line at the bottom of the left panel depicts the intensity according to the Ernst formula for $E_{2}=0$ or for pure longitudinal interference without refocusing of transversal components. For certain quadratic increments $c$ (some manufacturers use $50^{\circ}$ or $117^{\circ}$ ). the "rf-spoiled" signal will approximate this value, but it may be even less than the Ernst amplitude.

One goal in the design of robust rf-spoiled sequences is to achieve a consistent $T_{1}$ contrast for all relevant $T_{1}, T_{2}$, and $\alpha$ values. The gray value coded image on the right panel in Fig. 10 shows the rf-spoiled steady-state amplitude of the $F_{0}^{+}$signal $\left(\alpha=60^{\circ}\right)$ as a function of $T_{2}\left(T_{1}=50 T_{R}\right)$ and the quadratic increment $c$. For certain values of $c$, $F_{0}^{+}$is consistently close to the ideal spoiled Ernst value, independent of $T_{2}$.

It is very interesting and informative to look at the evolution of the $F_{n}$ configurations during an rf-spoiled sequence. Figure 11 displays the boomerang-shaped creation of $F_{n}$ states during rf excitation for different quadratic phase increments $c$. In contrast to the example shown in Fig. 7 , the $F_{n}$ configurations are forced to travel back toward the center, where they interact with the dominating, central $F_{0}$ state. After several hundreds of excitation pulses, they destructively contribute to the center line and cause a cancellation of steady-state transversal components. The numerical simulations shown in Figs. 7, 10, and 11 are based on the $\mathrm{C}$ program given in the Appendix.

\section{Completely Balanced Sequences: TRUE FISP}

In all previous discussions and examples, we assumed that the transversal magnetization will dephase between rf pulses owing to the applied readout gradient. The dephasing is necessary to read out the fully encoded echo, but it can be rephased by a reversed gradient pulse into pure magnetization, as depicted in the diagram in Fig. 12. This type of sequence was originally called FISP (14), but was not in clinical use for several years owing to high requirements on field homogeneity and gradient performance. It has now been implemented on state-of-the-art scan-
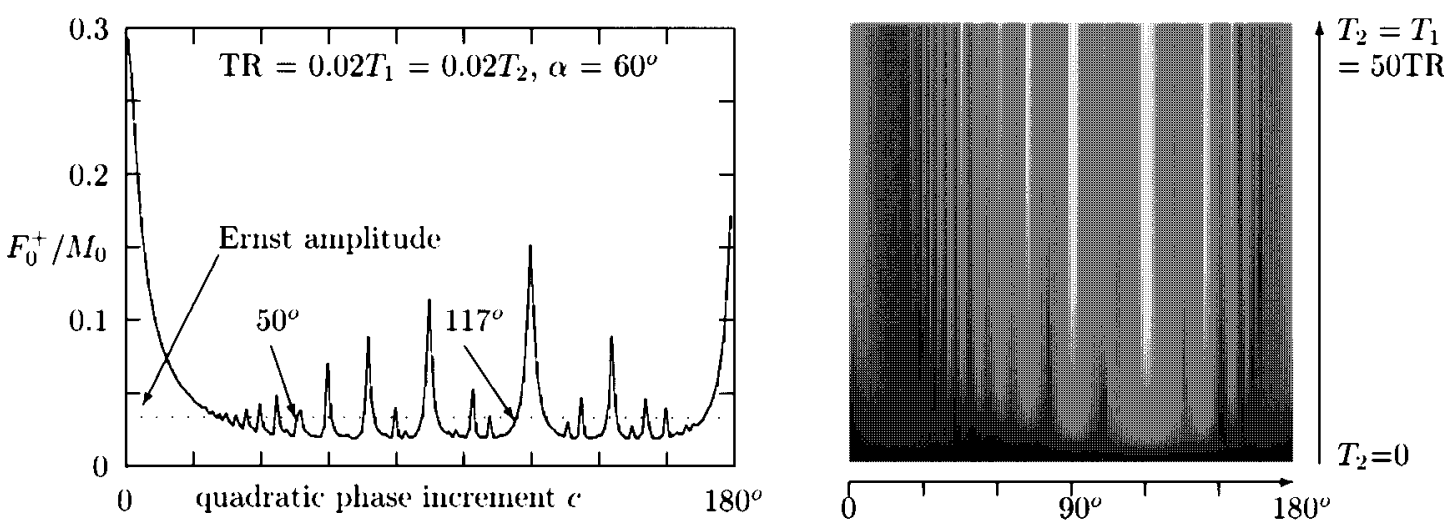

Figure 10 Steady-state $F_{0}^{+}$amplitude of an rf-spoiled sequence as a function of the quadratic rf pulse phase increment $c$. The dotted line depicts the Ernst amplitude (pure FID signal) for the parameters $T_{1}=T_{2}=50 T_{R}$ and rf flip angle $\alpha=60^{\circ}$. The right panel is a grayvalue coded plot of the steady-state amplitude as a function of the phase increment $c$ and $T_{2}\left(T_{1}=50 T_{R}\right)$. The spoiled steady-state amplitude obviously depends on $T_{2}$ and $T_{1}$ but is approximately constant for different quadratic phase increments such as $50^{\circ}, 80^{\circ}, 103^{\circ}, 117^{\circ}$, $124^{\circ}$, or $142^{\circ}$. 


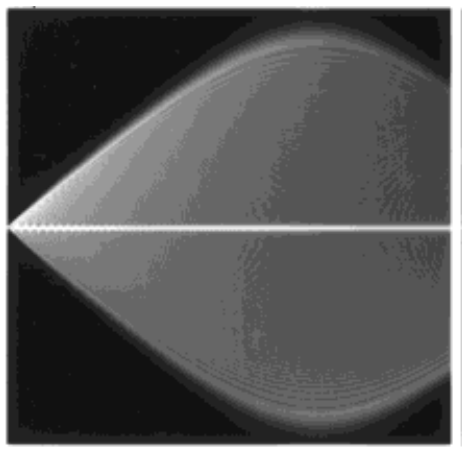

$\mathrm{c}=2^{\circ}$

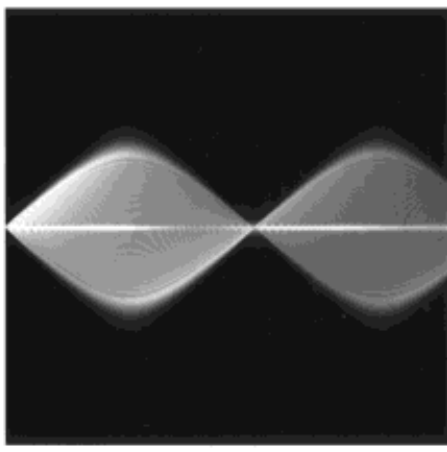

$\mathrm{c}=5^{\circ}$

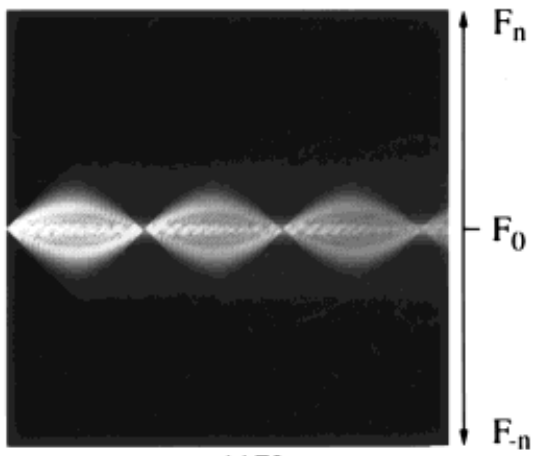

$\mathrm{c}=117^{\circ}$

Figure 11 Evolution of transversal $F_{n}$ states during an rf-spoiled sequence. In contrast to constant or linear rf pulse phases, the peripheral wave returns back to the central $F_{0}$ line and starts a sinusodial oscillation of spreading and confluence. The oscillation frequency increases with increased quadratic phase increment $c$.
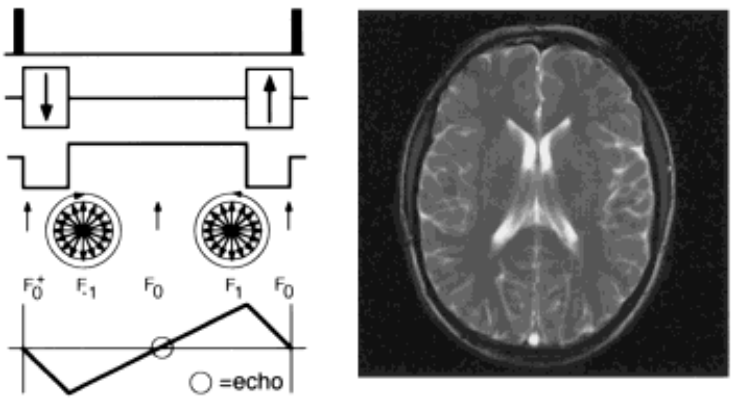

Figure 12 Completely balanced protocol of the TRUE FISP sequence (14). Both phase-encoding and readout gradients are rephased between rf pulses and no higherorder dephased $F_{n}$ states are being generated. The sequence offers a very high signal-to-noise ratio and shows essentially a $T_{1} / T_{2}$ contrast.

ners and renamed "TRUE FISP." The $T_{2}$-like contrast and an excellent signal-to-noise ratio makes it useful for several clinical applications as shown, for example, in Fig. 1.

The most remarkable point is that beside the $F_{0}$ state, no higher-order $F_{n}$ states will be created, at least in the ideal case. The resultant phase-graph shown in Fig. 12 consists of a single zigzag line and no configurations with higher dephasing are being formed. It is important to use alternating $\mathrm{rf}$ pulses for excitation (i.e., linear phase increment of $\left.\phi_{n}=n \pi\right)$ to avoid the refocused signal having an opposed phase to the primary echo. An image slice positioned identical to all previous examples is shown in the right panel of Fig. 12.

The signal intensity as a function of $T_{1}, T_{2}$, and flip angle $\alpha$ can be derived from Eq. [14] and is given by (13)

$$
F_{0}=\frac{\left(1-E_{1}\right) \sqrt{E_{2}} \sin \alpha}{1-\left(E_{1}-E_{2}\right) \cos \alpha-E_{1} E_{2}}
$$

if the echo is centered between the rf pulses. For liquids where $T_{1} \sim T_{2}$ and for a repetition time $T_{R} \leq T_{1}$, the amplitude of $F_{0}$ reaches the value of $0.5 M_{0}$, which is much higher than previous steadystate sequences.

The completely balanced imaging protocol of TRUE FISP is very sensitive to field inhomogeneities, susceptibility effects, and varying eddy currents induced by the stepped phase-encoding gradient. Some authors claim that this sequence is not able to generate artifact-free images on clinical scanners $(13,16,17)$, which is certainly not true even on high field systems. The crucial point in TRUE FISP imaging is that the $F_{0}$ state consists of a superposition of the primary echo and of several rf pulse-rephased states that probably show slight phase differences caused by gradient imperfections or field inhomogeneities. High-performance gradient systems (rise times of about $50 \mathrm{~T} / \mathrm{m} / \mathrm{s}$ ) are able to reduce the repetition time $T_{R}$ to $5 \mathrm{~ms}$ for a pixel resolution of $1 \times 1$ $\mathrm{mm}$, and appropriate shimming can reduce the residual field variation to $1 \mathrm{ppm}$. Phase differences between consecutive rf pulses can therefore be minimized and a homogeneous intensity across the entire image slice can be obtained, as shown in Figs. 1 and 12.

\section{SUMMARY}

The interaction between spins, rf fields, and gradient fields can be described by the classical Bloch equations. Both the rf field and gradient field simply rotate the magnetization vector of the spin ensemble. A consecutive application of these rotations can create the described range 
of different mechanisms of echo formation. It was demonstrated, however, that the description of multipulse imaging experiments based on isolated magnetization vectors, and Bloch rotations is not adequate for a pictorial understanding of the stimulated echo. It is also by no means able to illustrate the appearance of all higher-order echoes.

The basic concept was to substitute the common single-spin vector model for the benefit of a more appropriate description of magnetization. The introduction of dephased states with zero net magnetization as a fundamental building block of multipulse experiments enabled a universal and clearly arranged definition of the instantaneous configuration of the magnetization. The transition and flow between different magnetization states is controlled by the transition matrix (the complex Bloch equation applied to dephased states) and the timing or phase evolution between rf pulses. The resulting configuration and superposition of different magnetization states were used to calculate the echo amplitude, and thus the image contrast. The most practical and delightful approach to calculate and display the creation of magnetization states is a numerical simulation of an imaging sequence based on Eqs. [7] and [8]. Some results have been shown in Figs. 7, 10, and 11 , and the reader is encouraged to simulate the destiny of magnetization states for more sophisticated imaging techniques such as nonrefocused FLASH sequences, multiecho BURST sequences, or rf-spoiled projection sequences. The C program listed in the Appendix may be helpful to transfer the relevant equations into an executable code.

\section{APPENDIX}

\section{Numerical Simulation of Steady States}

The $\mathrm{C}$ program listed below simulates the evolution of transversal and longitudinal states for a multipulse experiment with constant rf pulse spacing $T_{R}$ and constant dephasing. The complex transversal states $F_{n}$ and $F_{n}^{+}$are stored as $(\mathrm{fx}[\mathrm{np}+\mathrm{n}], \mathrm{fy}[\mathrm{np}+\mathrm{n}])$ and $(\mathrm{pfx}[\mathrm{np}+\mathrm{n}]$, pfy $[n p+n])$ for $n=-n p \cdots n p-1$. The $Z_{n}$ and $Z_{n}^{+}$states are given by $(\mathrm{zx}[\mathrm{n}], \mathrm{zy}[\mathrm{n}])$ and (pzx $[n], p z y[n])$ for $n=0 \cdots n p-1$. The program prints the $F_{0}^{+}$and $F_{-1}$ states after each rf pulse. The $T_{1}, T_{2}$, and $\mathrm{rf}$ pulse parameters correspond to the simulation shown in Fig. 7.

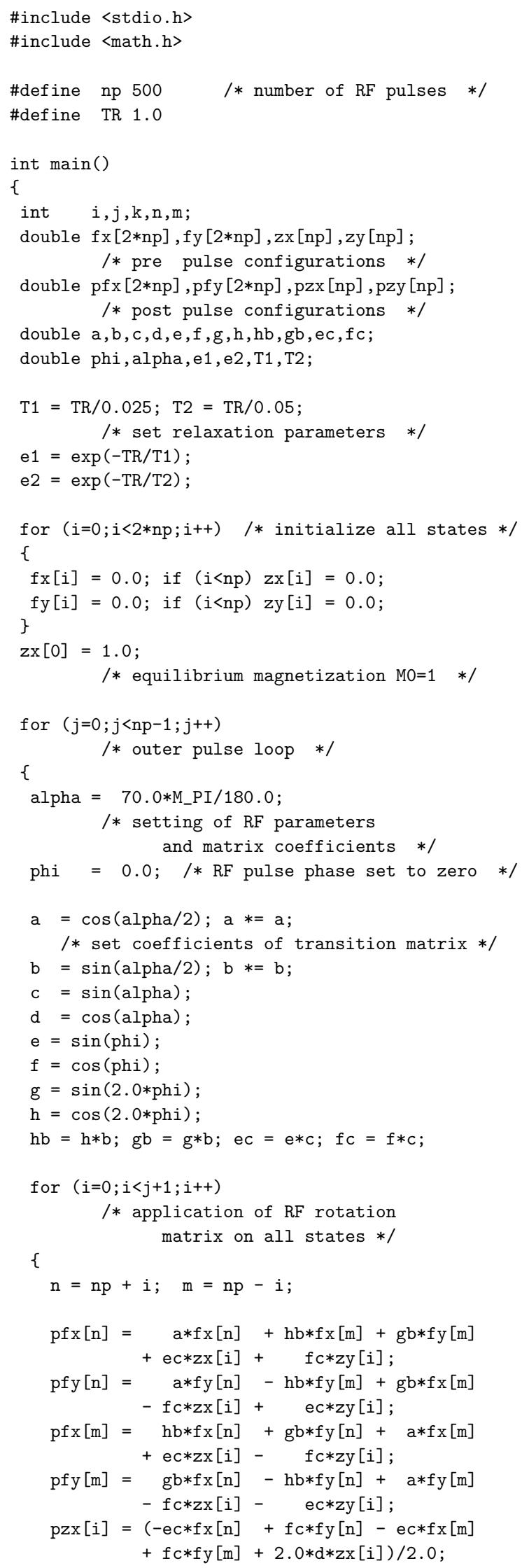




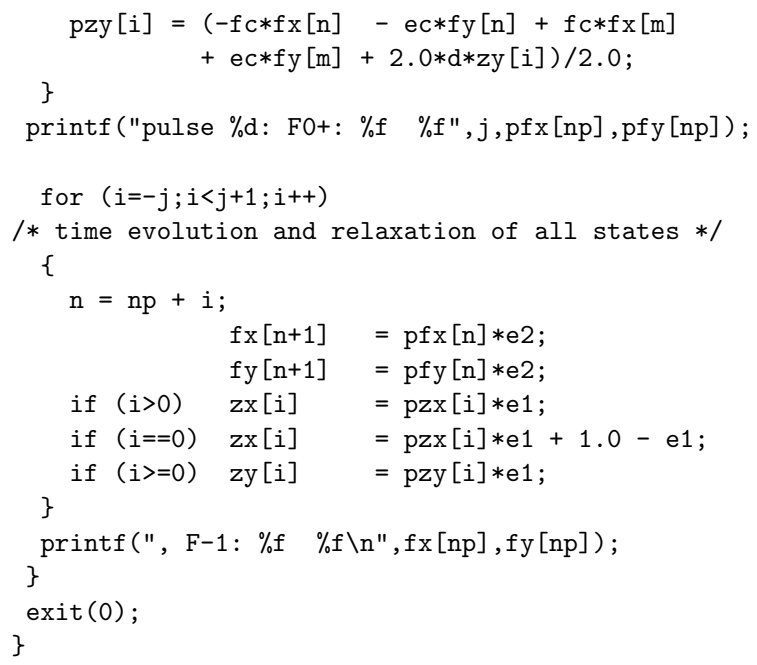

\section{ACKNOWLEDGMENTS}

The author acknowledges the considerable contributions to this field by current collaborators, particularly Reta Haselhorst, Oliver Heid, Jürgen Hennig, and Joachim Seelig. He is also grateful for financial support by the Swiss National Science Foundation under Grant 31-49758.96

\section{REFERENCES}

1. Ernst RR, Bodenhausen G, Wokaun A. Principles of nuclear magnetic resonance in one and two dimensions. Oxford: Clarendon; 1987. p 125-148.

2. Hahn EL. Spin echoes. Phys Rev 1950; 89: 580-594.

3. Woessner DE. Effects of diffusion in nuclear magnetic resonance spin-echo experiments. J Chem Phys 1961; 34: 2057-2061.

4. Kaiser R, Bartholdi E, Ernst RR. Diffusion and field-gradient effects in NMR Fourier spectroscopy. J Chem Phys 1974; 60: 2966-2979.

5. Hennig J. Multiecho imaging sequences with low refocusing flip angles. J Magn Reson 1988; 78: 397 407.

6. Frahm J, Merboldt KD, Hänicke W, Haase A. Stimulated echo imaging. J Magn Reson 1985; 64: 8193.

7. Hennig J. Generalized MR interferography. Magn Reson Med 1990; 16: 390-402.

8. Jaynes ET. Matrix treatment of nuclear induction. Phys Rev 1955; 98: 1099.

9. Gyngell ML, Palmer ND, Eastwood LM. The application of steady-state free precession (SFP) in 2D-FT MR imaging. In: Proceedings, SMRM, 5th annual meeting; 1986. p 666.

10. Hawkes RC, Patz S. Rapid Fourier imaging using steady-state free precession. Magn Reson Med 1987; 4: 9-23.

11. Gyngell ML. The steady-states signals in shortrepetition-time sequences. J Magn Reson 1989; 81: 474-483.

12. Mizumoto CT, Yoshitome E. Multiple echo SSFP sequences. Magn Reson Med 1991; 18: 244-250.

13. Zur Y, Stokar S, Bendel P. An analysis of fast imaging sequences with steady-state transverse magnetization refocusing. Magn Reson Med 1988; 6: 175193.

14. Oppelt A, Graumann R, BarfußH, Fischer H, Hartl W, Schajor W. FISP: eine neue schnelle Pulssequenz für die Kernspintomographie. Electromedica 1986; 54: $15-18$.

15. Hennig J. Echoes-how to generate, recognize, use or avoid them in MR-imaging sequneces: Part 1. Concepts Magn Reson 1991; 3: 125-143.

16. Hennig J. Echoes-how to generate, recognize, use or avoid them in MR-imaging sequneces: Part 2. Concepts Magn Reson 1991; 3: 179-192.

17. Sobol WT, Gauntt DM. On the stationary states in gradient echo imaging. J Magn Reson Imag 1996; 6: 384-398.

18. Meiboom S, Gill D. Modified spin-echo method for measuring nuclear relaxation times. Rev Sci Instrum 1958; 29: 688.

19. Heid O, Deimling M. QUEST—a quick echo split imaging technique. In: Proceedings, SMRM, 11th annual meeting; 1992. p 433.

20. Mugler JP, Epstein FH, Brookeman JR. Shaping the signal response during the approach to steadystate in three-dimensinal magnetization-prepared rapid gradient-echo imaging using variable flip angles. Magn Reson Med 1992; 28: 165-185.

21. Zur Y, Wood ML, Neuringer LJ. Spoiling of transverse magnetization in steady-state sequences. Magn Reson Med 1991; 21: 251-263.

22. Freeman R, Hill HDW. Phase and intensity anomalies in Fourier transform NMR. J Magn Reson 1971; 4: 366-383.

23. Ernst RR, Anderson WA. Application of Fourier transformation spectroscopy to magnetic resonance. Rev Sci Instrum 1996; 37: 93-102.

24. Hinshaw WS. Image formation by nuclear magnetic resonance: The sensitive point method. J Appl Phys 1976; 47: 3709-3721.

25. Heid O. Die $T_{1}$-gewichtete zerebrale KernspinSerienangiotomographie. MD thesis, University of Bern, Switzerland, 1998.

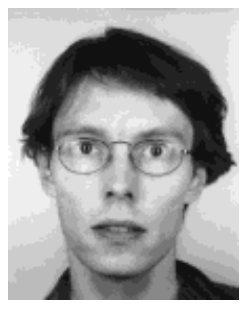

metallurgy.
Klaus Scheffler received his diploma in theoretical physics from the University of Freiburg, Germany, and his Ph.D. in Biophysical Chemistry from the University of Basel, Switzerland, in 1995. $\mathrm{He}$ is currently a staff associate at the University Hospital of Freiburg, Germany. His research interests include stochastic imaging, ultrafast imaging, and 